\title{
Easy collective polarization switching in ferroelectrics
}

\author{
A.M. Bratkovsky ${ }^{1}$ and A.P. Levanyuk ${ }^{1,2}$ \\ ${ }^{1}$ Hewlett-Packard Laboratories, 1501 Page Mill Road, Palo Alto, California 94304 \\ ${ }^{2}$ Departamento de Física de la Materia Condensada, C-III, Universidad Autónoma de Madrid, 28049 Madrid, Spain
}

(May 18, 2000)

\begin{abstract}
The actual mechanism of polarization switching in ferroelectrics remains a puzzle for many decades, since the usually estimated barrier for nucleation and growth is insurmountable ("paradox of the coercive field"). To analyze the mechanisms of the nucleation we consider the exactly solvable case of a ferroelectric film with a "dead" layer at the interface with electrodes. The classical nucleation is easier in this case but still impossible, since the calculated barrier is huge. We have found that the interaction between the nuclei is, however, long range, hence one has to study an ensemble of the nuclei. We show that there are the ensembles of small (embryonic) nuclei that grow without the barrier. We submit that the interaction between nuclei is the key point for solving the paradox.
\end{abstract}

77.80.Dj, 84.32Tt, 85.50.+k

The polarization switching in ferroelectrics (FEs) is most commonly used in applications (capacitors, memory elements), yet this process remains the least understood in spite of numerous experimental studies. As a rule, the ferroelectrics are switching in the field $E_{c}$ which is some order of magnitude lower than the so called "thermodynamic coercive field" $E_{c 0}$ [1 5 . The latter is the field at which the homogeneously polarized ferroelectric loses its stability in external field applied in opposite direction to the polarization. The fact that the switching takes place well before the point of instability is reached means that it proceeds by inhomogeneous nucleation and growth of domains of a new phase. But how the domains nucleate? The difficulty in answering this question has been emphasized by Landauer in late 1950s [1]. His and later estimates [2] 3] showed that the energy barrier for creating a nucleus with reversed polarization is practically insurmountable, $U^{*} \gtrsim 10^{3} k_{B} T$ in the field of about $100 \mathrm{kV} / \mathrm{cm}$. This problem, or a "paradox of a coercive field", has in fact been realized for domain nucleation in ferromagnets in 1938 [6]. The experimental coercive field for the bulk magnetic materials was known to be many times smaller than that suggested by the micromagnetics theory ("Brown's paradox") [7,8]. The disagreement is especially striking for hard magnetic materials, where the situation is closer to the ferroelectrics considered in the present paper. There were numerous suggestions over the years that some defects can assist the nucleation and reduce the coercive field down to experimentally observed values. The situation in magnetics was summarized by Brown in 1965 who noted that the idea is plausible but "there has been no strikingly successful calculation based on a completely realistic model" [7]. Since then the situation has apparently remained the same [8], whereas a switching in fine magnetic particles seems to be fairly described by micromagnetics theory.

The goal of the present paper is to suggest a possible new mechanism for the polarization switching in ferroelectric materials. To this end we consider an exactly solvable model of a ferroelectric material with an extended inhomogeneity of its dielectric response at the ferroelectric-electrode interface, which is called a "passive" or "dead" layer (for references see e.g. [9, 9, 10]).

The main feature of the ferroelectric film with the dead layer is that the film exists in a polydomain state at any thickness of the dead layer [9]. The monodomain state in this system, which can be produced by cooling in the field, would tend to transform into the equilibrium polydomain state. The transformation is favored by the fact that the polydomain state reduces the energy of the depolarizing field in spite of increased surface energy of the domain walls. This is contrary to the usual notion that the depolarizing field hinders the nucleation [1], yet the classical nucleation remains impossible.

First, we shall study the classical nucleation of the individual domains of the new phase. The nuclei will be assumed below to have a form of stripes or cylinders with the domain walls perpendicular to the plane of the film (c-domains), which is a reasonable approximation. We shall evaluate the barrier for their nucleation and show that for the individual nucleus it is practically insurmountable, although the dead layer helps to reduce it. We then abandon the classical approach and study the interaction between nuclei and find that it is long range.

It becomes clear that when an individual nucleus cannot grow the ensemble of nuclei may be able to. As an example of such an ensemble we consider a periodic array of nuclei and show that it indeed provides a path to the equilibrium state. We shall show that there is no energy barrier for its growth already when the nuclei become larger than the thickness of the domain wall $W$. After this 'embryonic' state has been passed the free energy of the system decreases monotonously as the nuclei grow. Obviously, the energy of embryonic nuclei is 
much smaller than the critical energy of the Landauer's nucleus [1]. This is simply related to the fact that the size of the critical Landauer needle-like nucleus is large, the radius of its base is $r_{c}^{\mathrm{L}}=W E_{c 0} / E_{\text {ext }} \gg W$, where $E_{\text {ext }}\left(\ll E_{c 0}\right)$ is the external field, therefore its energy $U_{\mathrm{L}}^{*}$ is huge. In the present case of the dead layer $E_{\text {ext }}$ should be replaced by the (small) depolarizing field in the ferroelectric and the critical radius of the Landauer's nucleus remains $\gg W$. Since for the collective nucleation $r_{c}^{\text {coll }} \sim W \ll r_{c}^{\mathrm{L}}$, the incurred energy barrier for a nuclei to grow beyond the embryonic state $U_{\text {coll }}^{*}$, if any, should be much smaller than the standard barrier $U_{\mathrm{L}}^{*}$ for individual nuclei. The equilibrium density of the embryos is large (see below) and the nucleation of the macroscopic domain (and the coercive field) would be determined by the waiting time for optimal fluctuation and its dependence on the electric field for an ensemble which can grow without, or almost without, the barrier,

We shall mainly discuss the energy aspects of the nucleation. What the present analysis demonstrates is that the nucleation of ferroelectric domains is facilitated by an interaction between the nuclei. Note that no such interaction is taken into account within the KolmogorovAvrami theory [11] which is widely used to treat switching in ferroelectrics.

We shall consider first the problem of the barrier for single stripe and then cylindrical domains, which appear to be insurmountable. We establish, however, that the interaction between the nuclei is long range and may give a clue to actual nucleation process. We then turn to exactly solvable case of an ensemble of stripe domains and show that the barrier for nucleation is actually zero already when the nuclei are at the embryonic stage.

The geometry of the present problem for ferroelectric with dead layer is illustrated in Fig. 1. For a shortcircuited electrodes (zero bias voltage) the free energy of the system is $\widetilde{F}=F_{0}+U_{e s}$, where the electrostatic energy $U_{e s}$ is [9]:

$$
U_{e s}=\frac{1}{2} \int_{\mathrm{FE}} \mathrm{d} \mathcal{A} \sigma \varphi
$$

where $\sigma$ is the bound charge due to the spontaneous polarization, $\varphi$ is the electrostatic potential, while $F_{0}$ includes the surface energy of the domain walls, and the integration goes over the FE surface. The electrostastic potential $\varphi$ is found from solving the Poisson equation for assumed domain structure [9]. We obtain with the use of the Fourier transformation the total electrostatic energy for arbitrary one-dimensional domain structure as

$$
U_{e s}=2 \int_{-\infty}^{\infty} \frac{\mathrm{d} k}{k} \frac{\left|\sigma_{k}\right|^{2}}{\sqrt{\varepsilon_{a} \varepsilon_{c}} \operatorname{coth}\left(\sqrt{\frac{\varepsilon_{a}}{\varepsilon_{c}}} \frac{k l}{2}\right)+\varepsilon_{g} \operatorname{coth} \frac{k d}{2}},
$$

where $\sigma_{k} \equiv \int_{-\infty}^{\infty} d x \exp (-i k x) \sigma(x, z=l / 2)$ is the Fourier component of the surface bound charge $\sigma(x, z=l / 2)$, $l$ the thickness of the FE film with $\varepsilon_{c(a)}$ the dielectric constants in $c(a)$ direction, $d$ the thickness of the dead layer (Fig. 1).

We begin with the case of a nucleus in the center of the plate, i.e. with the following distribution of the bound charge

$$
\begin{array}{llrl}
\sigma(x, z=+(-) l / 2) & =-(+) P_{s}, & & |x|<a / 2 ; \\
\sigma(x, z=+(-) l / 2)=+(-) P_{s}, & & a / 2<|x|<R,
\end{array}
$$

where $2 R$ is the width of the plate and $a$ is the width of the nucleus, and $P_{s}$ the spontaneous polarization. In this case $\sigma_{k}=\frac{2 P_{s}}{k}\left(-2 \sin \frac{k a}{2}+\sin k R\right)$. The dependence of all physical quantities on $R$ disappears in a limit $R \rightarrow \infty$, as it should. It is handy to always subtract the (constant) electrostatic energy of the uniformly polarized sample, which is characterized by the Fourier transform of the bound charge $\bar{\sigma}_{k}=\left(2 P_{s} / k\right) \sin k R$. By using Eq.(3) one then finds the change of the electrostatic energy due to creation of a stripe nucleus

$$
\begin{aligned}
U_{e s}^{\text {stripe }} & =-8 \pi \varepsilon_{g}^{-1} P_{s}^{2} d a\left[1-\frac{a}{\pi d} \ln \left(\frac{e^{3 / 2} d}{a}\right)\right], \quad a \lesssim d ; \\
& =-8 \varepsilon_{g}^{-1} P_{s}^{2} d^{2} \ln \left(\frac{e^{3 / 2} a}{d}\right), \quad a \gg d ;
\end{aligned}
$$

for $d<l \sqrt{\varepsilon_{c} / \varepsilon_{a}}, \varepsilon_{g}=\sqrt{\varepsilon_{a} \varepsilon_{c}}$. Note that this is the change in electrostatic energy with respect to uniformly polarized sample when the nucleus is present, so it does not apply to completely reversed sample. The electrostatic energy favors the nucleation, since the domains reduce the energy of stray field . The total energy of the stripe nucleus per unit length is

$$
\tilde{F}_{\text {stripe }}(a)=2 l \gamma+U_{e s}-2 P_{s} E_{\text {ext }} a l,
$$

where $\gamma=P_{s}^{2} \Delta$ is the surface energy of the domain wall with $\Delta$ the temperature dependent characteristic length [9]. We see that the gain in electrostatic energy eventually overwhelms the surface energy, and there appears an exponentially wide barrier for the nucleus in $\tilde{F}(a)$ when $E_{\text {ext }}=0$.

We shall now consider the case of two nuclei of a new phase in a form of stripe domains. With the help of Eq. (2) one can easily calculate the change of the electrostatic energy due to formation of two nuclei having the same width $a$, with the separation $r$ between their centers. We find for the energy of the interaction of two stripe domains per unit length for $r \gg a$

$$
U_{\text {int }}^{\text {stripe }}(r)=2 \varepsilon_{g}^{-1} P_{s}^{2} a^{2} d^{2} \frac{1}{r^{2}},
$$

which corresponds to long range dipole-dipole interaction between two stripes [12]. This observation indicates that the interactions in a system with an ensemble of nuclei would be very important. Note that the interaction of 
the stripe nucleus with the edge of the sample $U_{\text {edge }}$ is also long range, $U_{\text {edge }}\left(x_{0}\right)=2 \varepsilon_{g}^{-1} P_{s}^{2} a d^{2} / x_{0}$, and they are repelled from the edge.

Similar treatment can be repeated for a single and a pair of cylindrical nuclei. The expression for the electrostatic energy for cylindrical nucleus is similar to that for the stripe case (2)

$$
U_{e s}=2 \int_{0}^{\infty} \mathrm{d} k \frac{\left|\sigma_{k}\right|^{2}}{\sqrt{\varepsilon_{a} \varepsilon_{c}} \operatorname{coth}\left(\sqrt{\frac{\varepsilon_{a}}{\varepsilon_{c}}} \frac{k l}{2}\right)+\varepsilon_{g} \operatorname{coth} \frac{k d}{2}},
$$

where for the nucleus with the radius $a$ in the center of the slab with the radius $R$ we obtain $\sigma_{k} \equiv 2 \pi \int_{0}^{R} \mathrm{~d} r r J_{0}(k r) \sigma(r, z=l / 2)=$ $\left(2 \pi P_{s} / k\right)\left[R J_{1}(k R)-2 a J_{1}(k a)\right]$, while for the uniformly polarized sampe $\bar{\sigma}_{k}=\left(2 \pi P_{s} / k\right) R J_{1}(k R)$, with $J_{n}(z)$ the Bessel function. The integral in the expression (8) for the electrostatic energy of the cylindrical nucleus can be evaluated with the result

$$
\begin{aligned}
U_{e s}^{\text {cyl }} & =-8 \pi^{2} \varepsilon_{g}^{-1} P_{s}^{2} d a^{2}, \quad a \lesssim d ; \\
& =-8 \pi \varepsilon_{g}^{-1} P_{s}^{2} d^{2} a \ln \frac{8 a}{e^{1 / 2} d}, \quad a \gg d ;
\end{aligned}
$$

The free energy of one cylindrical nucleus is

$$
\tilde{F}_{\text {cyl }}(a)=2 \pi a l \gamma+U_{e s}-2 \pi P_{s} E_{\text {ext }} l a^{2} .
$$

It is obvious from Eqs. (9),(10) that the gain in the electrostatic energy eventually overwhelms the growth of the the surface energy of the domain wall with increase of the radius $a$ of the nucleus. The critical radius is exponentially large when $E_{\text {ext }}=0$, it behaves roughly as $a_{c} \sim d \exp \left(0.3 a_{K}^{2} / d^{2}\right)$, where $a_{K}=(0.3 \tilde{\varepsilon} \Delta l)^{1 / 2}$ is the Kittel period, $\tilde{\varepsilon}=\varepsilon_{g}+\sqrt{\varepsilon_{a} \varepsilon_{c}}$ [9]. The corresponding barrier height $\tilde{F}_{c}$ is very large, $\tilde{F}_{c} \gg P_{s}^{2} d_{a t}^{3} \sim E_{a t}$, where $d_{a t}$ is the characteristic "atomic" length (of the order of the lattice parameter) and the "atomic" energy $E_{a t}$ amounts to a few eV. Thus, in this case the barrier is also huge, comparable to the barrier for the Landauer's nucleus, and its growth is prohibitively expensive.

The interaction between two cylindrical nuclei can be estimated for $r \gg a$ as

$$
U_{i n t}^{\mathrm{cyl}}(r)=16 \pi^{2} \varepsilon_{g}^{-1} P_{s}^{2} a^{4} d^{2} \frac{1}{r^{3}}
$$

In this case it appears to also be long range of a dipoledipole type [12].

The importance of these interactions lies in their long range rather than sign. Therefore, one has to accurately evaluate the total energy for a system of nuclei, since it does not reduce to a sum of asymptotic interactions (7) or (11). The long range interactions give us a clue to the mechanism of nucleation and growth of a new phase. To illustrate that it indeed may solve the problem, we shall consider a system of stripe-like domains. The results below will demonstrate that the electrostatic energy not only favors nucleation but eliminates an energy barrier for the growth of nuclei in an ensemble when their size is larger than the domain wall width, i.e. the switching proceeds collectively.

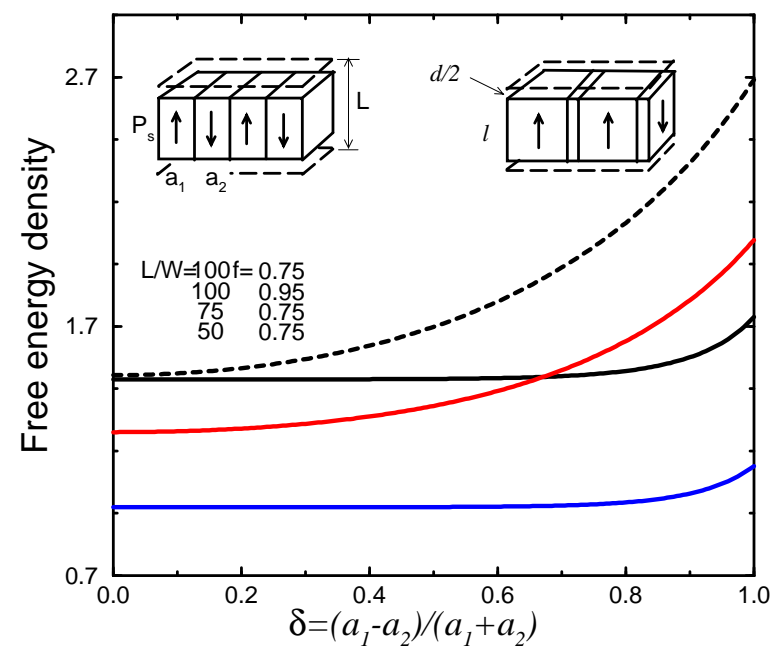

FIG. 1. Free energy density $\tilde{F} / \mathcal{A} P_{s}^{2}$ for the growth of ensemble of nuclei for different values $L / W$ and the fraction of volume occupied by the ferroelectric $f=l / L(d=L-l$ is the thickness of the dead layer). Note that there is no barrier for growth of very small nuclei (from $\delta \approx 1$ ) towards the domain pattern with equal width $\delta=0$ of the domains of both phases $P_{s}$ and $-P_{s}$ (Kittel state). The flatness of the free energy $\tilde{F}$ in the vicinity of the Kittel state demonstrates remarkable softness of the ferroelectric with the dead layer. Inset shows schematics of the growth of the ensemble of nuclei (narrow domains of the new phase).

To study the collective nucleation we shall analyze a domain structure with stripe domains of opposite polarization of widths $a_{1}$ and $a_{2}$, the period $T=a_{1}+a_{2}$, and the asymmetry parameter $\delta=\frac{a_{1}-a_{2}}{a_{1}+a_{2}}$, which measures a net polarization of the film [9]. For zero external bias voltage assumed throughout the present paper $\delta=0$ in the equilibrium (polydomain) state, whereas in the monodomain state $\delta=1$. At $\delta \rightarrow 1$ the system consists of very narrow domains with the polarization opposite to the net polarization (Fig. 1, inset), i.e. it is a periodic ensemble of nuclei. The free energy $\tilde{F}$ of this system at zero external bias is given by

$$
\begin{aligned}
\frac{\tilde{F}}{\mathcal{A} P_{s}^{2}}= & \frac{2 \pi d \delta^{2}}{\varepsilon_{c}(d / l)+\varepsilon_{g}}+\frac{2 \Delta l}{T}+\frac{16 T}{\pi^{2}} \sum_{j=0}^{\infty} \frac{1}{(2 j+1)^{3}} \frac{1}{D_{2 j+1}} \\
& +\frac{8 T}{\pi^{2}} \sum_{n=1}^{\infty}(-1)^{n} \frac{1-\cos \pi n \delta}{n^{3}} \frac{1}{D_{n}}
\end{aligned}
$$

where $D_{n}=\sqrt{\varepsilon_{a} \varepsilon_{c}} \operatorname{coth} \sqrt{\frac{\varepsilon_{a}}{\varepsilon_{c}}} \frac{\lambda_{n} l}{2}+\varepsilon_{g} \operatorname{coth} \frac{\lambda_{n} d}{2}$ and $\lambda_{n}=$ $2 \pi n / T$ [9]. Fortunately, this free energy can be found 
analytically in the asymptotic case of narrow dead layer $(d \ll l)$ and correspondingly wide period of the domain structure, $T \gg d$, when $\varepsilon_{g}=\sqrt{\varepsilon_{a} \varepsilon_{c}}$. We have found earlier that the period of the domain structure in this case is exponentially large, $T=0.95 d \exp \left(0.4 a_{K}^{2} / d^{2}\right)$. We note that in this case $D_{n}=\varepsilon_{g}\left(1+\operatorname{coth} \frac{\lambda_{n} d}{2}\right)$ and summation in (12) can be performed to yield

$$
\begin{aligned}
\frac{\tilde{F}}{\mathcal{A} P_{s}^{2}}= & \frac{2 \pi d \delta^{2}}{\varepsilon_{c}(d / l)+\varepsilon_{g}}+\frac{2 \Delta l}{T}+\frac{4 T}{\pi^{2} \varepsilon_{g}}\left[\zeta(3)-2 L i_{3}\left(e^{-b}\right)\right. \\
& +\frac{1}{4} L i_{3}\left(e^{-2 b}\right)-L i_{3}\left(-e^{-b}\right) \\
& \left.-\operatorname{Re} L i_{3}\left(-e^{i \pi \delta}\right)+\operatorname{Re} L i_{3}\left(-e^{i \pi \delta-b}\right)\right]
\end{aligned}
$$

where $b=4 \pi d / T \ll 1$, and $L i_{n}(z) \equiv \sum_{k=1}^{\infty} z^{k} / k^{n}$. For the case of an ensemble of narrow domains, $\delta \simeq 1$ the free energy can be found from the known asymptotic behavior of the $L i_{n}(z)$ function [13], yielding an approximate expression

$$
\frac{\tilde{F}}{\mathcal{A} P_{s}^{2}}=\frac{2 \pi d \delta^{2}}{\varepsilon_{c}(d / l)+\varepsilon_{g}}+\frac{2 \Delta l}{T}+\frac{T}{\varepsilon_{g}}(1-\delta)^{2} \ln \frac{e^{3} b^{2}}{\pi^{2}(1-\delta)^{2}} .
$$

Now everything depends on how the free energy $\tilde{F}$ behaves as a function of $\delta$ when the nuclei grow, i.e. when $\delta$ reduces from unity towards zero. One can easily see that at $x \equiv 1-\delta \ll 1$ the free energy $\tilde{F} / \mathcal{A} P_{s}^{2}$ is given, with respect to a constant, by the function

$$
f(x) \equiv-\frac{4 \pi d x}{\varepsilon_{c}(d / l)+\varepsilon_{g}}+\frac{T}{\varepsilon_{g}} x^{2} \ln \frac{e^{3} b^{2}}{\pi^{2} x^{2}},
$$

where $f(0)=0$. This function does not have a barrier as a function of $x$ when $T$ is kept constant. Indeed, the second term in (15) has an exponentially small maximum $\left(=T \varepsilon_{g}^{-1} x_{0}^{2}\right)$ at $x_{0}=4 e d / T \ll 1$. It is, however, suppressed by the first term, which corresponds to the energy of homogeneous field created by the net polarization and is linear in $x$. As a result, there appears to be no barrier for the growth of nuclei. Note that we have actually restricted the system's path for nucleation by constraining the domain pattern to a fixed period. Even under this constraint the growth proceeds without the energy barrier, and this would be even more so if we were to lift the constraint and allow the system to follow an optimal path to equilibrium. This behavior does not depend on the approximation we have made for evaluating the free energy, the exact calculation of the free energy (12) for all $\delta$ shows that the collective growth of nuclei we just described proceeds without the barrier (Fig. 1). As mentioned above, the smallest size of nuclei where the present analysis applies is of the order of the domain wall width $W$ and the barrier for the nucleation of such small embryonic nuclei is expected to be zero or much smaller than the usual estimates [1] 3] for individual nucleation.
For nucleation to proceed by the present mechanism the only condition is the presence of extended dielectric inhomogeneity in a sample. The external field does promote growth of the nuclei, but the nucleation in the present system occurs even without it. The likely requirement is that the lateral extent of this inhomogeneity should be much larger than the period of the equilibrium domain (Kittel) structure $a_{K}$. One can estimate the rate of embryo nucleation in $1 \mathrm{~cm}^{3}$ as $\sim\left(1 / W^{3} \tau_{p h}\right) \exp \left(-U_{e m} / k T\right)$, where $\tau_{p h}$ is the characteristic (optical phonon) time, and the "atomic" estimate of the energy of the embryo is $U_{e m} \sim \gamma W^{2} \sim$ $E_{a t} \sqrt{T_{c} / T_{a t}} \sim 2-3 \cdot 10^{3} \mathrm{~K}$ [14], with the characteristic "atomic" temperature $T_{a t} \sim 10^{4} \mathrm{~K}$. Taking a conservative estimate of the embryo lifetime as $\sim 10 \tau_{p h}$, one finds the equilibrium density of the embryos $\sim 10^{17} \mathrm{~cm}^{-3}$. At such high densities the embryos should 'feel' the field of each other, and favorable ensembles should appear within a reasonable time, unlike in the case of the Landauer's nucleus where the expectation time much exceeds the lifetime of the universe.

In conclusion, we have suggested a possible way to solve the "paradox of the coercive field" by demonstrating a collective mode of domain growth past the embryonic stage with sizes about the domain wall thickness $W$, which proceeds without energy barrier. The origin of this cooperative phenomenon is a long-range interaction of electrostatic origin between the nuclei. The possible screening by free charges in ferroelectric does not seem to be important, since the conductivity of ferroelectrics is usually too low to have any effect [1]. As a corollary, we note that the Kolmogorov-Avrami model (KA) 11 is inapplicable to growth of domains in ferroelectrics, since the essential long-range interaction between nuclei is completely neglected in this approach (note that KA fully bypasses the question of how the domains were nucleated in the first place). The present results are general, and have the implication that in ferroelastic materials and possibly also magnetic materials the nucleation would be facilitated by the long-range interaction between nuclei of a new phase. This could alleviate the Brown's paradox of the coercive field in relation to switching in bulk ferromagnets.

We acknowledge helpful discussions with A. Aharoni, V.V. Osipov, A.L. Roytburd, and A.K. Tagantsev.

[1] R. Landauer, J. Appl. Phys. 28, 227 (1957).

[2] V. Janovec, Czech. J. Phys. 8, 3 (1958).

[3] H.F. Kay and J.W. Dunn, Phil. Mag. 7, 2027 (1962).

[4] V.V. Lemanov and V.K. Yarmarkin, Phys. Sol. State 38, 1363 (1996). 
[5] J.F. Scott, Ferroelecrics 183, 51 (1996).

[6] W. Döring, Z. Physik 108, 137 (1938).

[7] W.F. Brown Jr., in Fluctuation Phenomena in Solids, edited by R.E. Burgess (Academic, New York, 1965), Ch. 2, p. 37.

[8] A. Aharoni, Introduction to the Theory of Ferromagnetism (Oxford, England, 1996), Ch. 9.

[9] A.M. Bratkovsky and A.P. Levanyuk, Phys. Rev. Lett. 84, 3177 (2000).

[10] For ferroelastics analogue, see e.g. A.L. Roytburd, J. Appl. Phys. 83, 228, 239 (1998).

[11] A.N. Kolmogorov, Izv. Akad. Nauk. USSR., Ser. Math. 3, 355 (1937); M. Avrami, J. Chem. Phys. 7, 1103 (1939); 8, 212 (1940); 9, 177 (1941).

[12] Note that the stripe domain and its mirror image form the dipole moment $2 P_{s} a d$ (per unit length). For cylindrical nucleus it equals $2 P_{s} \pi a^{2} d$. The coefficients in Eqs. (7), (11) reflect the interaction between infinite stacks of the mirror images.

[13] $L i_{n}(z)=z \Phi(z, n, 1)$, where $\Phi(z, n, v)$ is the Lerch function: see e.g. I.S. Gradshteyn and I.M. Ryzhik, Table of Integrals, Series, and Products, 5th ed., edited by A.Jeffrey (Academic, New York, 1994), Sec. 9.55.

[14] At these small sizes it does not make sense to consider a particular geometry of embryos, so we make the order of magnitude estimates. 